\title{
MEMBRANE CAPS*
}

By

\author{
R. W. DICKEY
}

University of Wisconsin, Madison

\begin{abstract}
The exact theory for the rotationally symmetric deformation of a membrane cap under either a gravitational force or a normal force is derived. It is shown that in either case the exact theory can be reduced to a single, second-order, ordinary differential equation for the determination of a quantity related to the radial stress. This equation is specialized to the small strain case. Approximate theories are obtained from the exact equations. In particular, the correct approximate equations are obtained when the applied pressure is small and when the membrane cap is shallow. The shallow spherical cap is treated as a special example.
\end{abstract}

1. Introduction. The purpose of this paper is to discuss the rotationally symmetric deformation of membrane caps. Approximate nonlinear theories for membrane caps have been described by Bromberg and Stoker [1] and Reissner [2]. The spherical cap is a configuration of special interest, and approximate solutions have been found using asymptotic methods (cf. [1]) and numerical methods (cf. [3]).

In this paper we will discuss the exact theory of membrane caps. It is convenient to formulate the equations in cylindrical coordinates (cf. Sec. 6). Thus we denote the unit vectors in the $r, \boldsymbol{\theta}$, and $z$ direction by $\mathbf{e}_{r}, \mathbf{e}_{\theta}$, and $\mathbf{k}$. In rectangular coordinates the vectors are

$$
\mathbf{e}_{r}=\cos \theta \mathbf{i}+\sin \theta \mathbf{j}, \quad \mathbf{e}_{\theta}=-\sin \theta \mathbf{i}+\cos \theta \mathbf{j},
$$

where $\mathbf{i}$ and $\mathbf{j}$ are the unit vectors in the $x$ and $y$ directions. If the membrane undergoes an axially symmetric deformation, a point whose position on the undeformed surface is given by

$$
\mathbf{R}=r \mathbf{e}_{r}+Z(r) \mathbf{k}
$$

*Received September 29, 1986. 
will have a new position

$$
\mathbf{R}^{*}=(r+u(r)) \mathbf{e}_{r}+(z(r)+w(r)) \mathbf{k}
$$

after the deformation. $u$ and $w$ are the displacements in the radial and $z$ directions. The radial and circumferential strains which arise from the deformation described by (1.2) and (1.3) are

$$
\begin{aligned}
\varepsilon_{r} & =\frac{u^{\prime}+z^{\prime} w^{\prime}+\frac{1}{2}\left(u^{\prime}\right)^{2}+\frac{1}{2}\left(w^{\prime}\right)^{2}}{m^{2}}, \\
\varepsilon_{\theta} & =\frac{u}{r}+\frac{1}{2} \frac{u^{2}}{r^{2}},
\end{aligned}
$$

where

$$
m^{2}=1+\left(z^{\prime}\right)^{2}
$$

$\left({ }^{\prime}=d / d r\right)$. The equilibrium equations are:

$$
\begin{gathered}
\frac{d}{d r}\left\{\frac{\sigma_{r}(r+u)\left(1+u^{\prime}\right)}{\sqrt{\left(1+u^{\prime}\right)^{2}+\left(z^{\prime}+w^{\prime}\right)^{2}}}\right\}-\sigma_{\theta} \sqrt{\left(1+u^{\prime}\right)^{2}+\left(z^{\prime}+w^{\prime}\right)^{2}}+\frac{P_{1}}{h}=0, \\
\frac{d}{d r}\left\{\frac{\sigma_{r}(r+u)\left(z^{\prime}+w^{\prime}\right)}{\sqrt{\left(1+u^{\prime}\right)^{2}+\left(z^{\prime}+w^{\prime}\right)^{2}}}\right\}+\frac{P_{2}}{h}=0,
\end{gathered}
$$

where $\sigma_{r}$ and $\sigma_{\theta}$ are the radial and circumferential stresses and $P_{1}$ and $P_{2}$ are the components of applied force per unit undeformed area in the $r$ and $z$ directions. We will treat two cases of interest. If the force is in the $z$ direction (e.g. gravity) then

$$
P_{1}=0, \quad P_{2}=r m P(r) .
$$

If the force is in the direction normal to the undeformed surface (this is the assumption of the Bromberg-Stoker theory) then

$$
P_{1}=-r z^{\prime} P(r), \quad P_{2}=r P(r) .
$$

In both (1.7) and (1.8) $P(r)$ is the prescribed force per unit undeformed area. We note that in the case of the plane membrane $\left(z^{\prime}=0, m=1\right)$ equations (1.7) and (1.8) reduce to the same equations. The system of equations (1.4) and (1.6) is completed by two constitutive laws. In their general form they can be written

$$
\begin{aligned}
& \sigma_{r}=\sigma_{r}\left(\varepsilon_{r}, \varepsilon_{\theta}\right), \\
& \sigma_{\theta}=\sigma_{\theta}\left(\varepsilon_{r}, \varepsilon_{\theta}\right) .
\end{aligned}
$$

If the strains are small equations (1.9) can be taken as Hooke's law,

$$
\begin{aligned}
& \sigma_{r}=\frac{E}{1-\nu^{2}}\left(\varepsilon_{r}+\nu \varepsilon_{\theta}\right), \\
& \sigma_{\theta}=\frac{E}{1-\nu^{2}}\left(\varepsilon_{\theta}+\nu \varepsilon_{r}\right)
\end{aligned}
$$

where $E$ and $\nu$ are the Young's modulus and Poisson ratio. 
The boundary conditions to be prescribed on the equations (1.4), (1.6), and (1.9) are

$$
u(0)=w^{\prime}(0)=w(a)=0,
$$

where $a$ is the radius of the base of the undeformed membrane. In addition we prescribe either the radial displacement at the boundary (displacement condition)

$$
u(a)=\mu,
$$

or the radial stress (stress condition)

$$
\sigma_{r}(a)=\sigma
$$

In [4] it was shown that the exact equations for the rotationally symmetric deformation of a plane circular membrane could be reduced to a single, second-order, ordinary differential equation for the determination of a quantity related to the radial stress. In Section 2 it will be shown that a similar result holds for equations (1.4), (1.6), and (1.9). In Section 3 the small strain theory is obtained as a special case of the exact theory described in Section 2. In Sections 4 and 5 the small strain theory is discussed for both the gravitational case (cf. (1.7)) and the normal pressure case (cf. (1.8)).

The plane membrane is of course a special case of the membrane cap. It is shown that the theory for membrane caps does reduce to the theory for plane circular membranes described in [4]. In addition the relation between the exact theory for the plane circular membrane and the approximate theories due to Föppl [5] and to Reissner [6,7] are described.

In Sections 4 and 5 the appropriate shallow cap approximations are obtained in both the gravitational case and normal pressure case and the spherical cap is treated as a case of special interest. In the appendix (Section 6) the equations (1.4) and (1.6) are derived.

2. Reduction of the equations. In this section it will be shown that the equations (1.4), (1.6), and (1.9) can be reduced to a single equation. For this purpose it is convenient to rewrite equations (1.4) in the form

$$
\begin{gathered}
\sqrt{\left(1+u^{\prime}\right)^{2}+\left(z^{\prime}+w^{\prime}\right)^{2}}=m \sqrt{2 \varepsilon_{r}+1}, \\
r+u=r \sqrt{2 \varepsilon_{\theta}+1} .
\end{gathered}
$$

It is a consequence of (2.1) that the equilibrium equations (1.6) can be written

$$
\begin{gathered}
\frac{d}{d r}\left\{\frac{r \Sigma_{r}\left(1+u^{\prime}\right)}{m \sqrt{2 \varepsilon_{r}+1}}\right\}-m \Sigma_{\theta}+\frac{P_{1}}{h}=0, \\
\frac{d}{d r}\left\{\frac{r \Sigma_{r}\left(z^{\prime}+w^{\prime}\right)}{m \sqrt{2 \varepsilon_{r}+1}}\right\}+\frac{P_{2}}{h}=0,
\end{gathered}
$$

where

$$
\Sigma_{r}=\sigma_{r} \sqrt{2 \varepsilon_{\theta}+1}, \quad \Sigma_{\theta}=\sigma_{\theta} \sqrt{2 \varepsilon_{r}+1} .
$$


After integration equations (2.2) become

$$
\begin{gathered}
\frac{r \sum_{r}\left(1+u^{\prime}\right)}{m \sqrt{2 \varepsilon_{r}+1}}=r S-r F_{1}, \\
\frac{r \Sigma_{r}\left(z^{\prime}+w^{\prime}\right)}{m \sqrt{\left(2 \varepsilon_{r}+1\right)}}=-r F_{2},
\end{gathered}
$$

where

$$
r S=\int_{0}^{r} m \Sigma_{\theta} d \tau
$$

and

$$
r F_{1}=\frac{1}{h} \int_{0}^{r} P_{1}(\tau) d \tau, \quad r F_{2}=\frac{1}{h} \int_{0}^{r} P_{2}(\tau) d \tau
$$

Both $F_{1}$ and $F_{2}$ are known functions (cf. (1.7) or (1.8)). Equations (2.4) imply that

$$
r^{2} \Sigma_{r}^{2}=r^{2} F_{2}^{2}+r^{2}\left(S-F_{1}\right)^{2} \text {. }
$$

Combining (2.7) and (2.4) we find

$$
\begin{aligned}
& \Sigma_{r}=\sqrt{F_{2}^{2}+\left(S-F_{1}\right)^{2}}, \\
& z^{\prime}+w^{\prime}=-\frac{F_{2}\left(1+u^{\prime}\right)}{S-F_{1}} .
\end{aligned}
$$

It follows from (2.1a) and (2.8b) that

$$
1+u^{\prime}=\frac{m \sqrt{2 \varepsilon_{r}+1}\left(S-F_{1}\right)}{\Sigma_{r}} .
$$

It is a consequence of (2.5) that

$$
\Sigma_{\theta}=\frac{(r S)^{\prime}}{m}
$$

Equation (2.1b) implies that

$$
1+u^{\prime}=\frac{d}{d r}\left(r \sqrt{2_{\theta}+1}\right)
$$

so that (cf. (2.9))

$$
\frac{d}{d r}\left(r \sqrt{2 \varepsilon_{\theta}+1}\right)=\frac{m \sqrt{2 \varepsilon_{r}+1}\left(S-F_{1}\right)}{\Sigma_{r}} .
$$

The functions $\varepsilon_{r}$ and $\varepsilon_{\theta}$ in (2.12) are determined by the constitutive laws (1.9) which can be written

$$
\frac{\Sigma_{r}}{\sqrt{2 \varepsilon_{\theta}+1}}=\sigma_{r}\left(\varepsilon_{r}, \varepsilon_{\theta}\right), \quad \frac{\Sigma_{\theta}}{\sqrt{2 \varepsilon_{r}+1}}=\sigma_{\theta}\left(\varepsilon_{r}, \varepsilon_{\theta}\right)
$$


We will assume that the equations (2.13) have a unique solution for $\varepsilon_{r}$ and $\varepsilon_{\theta}$ as functions of $\Sigma_{r}$ and $\Sigma_{\theta}$, i.e.,

$$
\varepsilon_{r}=\varepsilon_{r}\left(\Sigma_{r}, \Sigma_{\theta}\right), \quad \varepsilon_{\theta}=\varepsilon_{\theta}\left(\Sigma_{r}, \Sigma_{\theta}\right)
$$

This is certainly the case for small strains where Hooke's law applies. Since $\Sigma_{r}$ and $\Sigma_{\theta}$ are known functions of $S$ an'd $S^{\prime}$ (cf. (2.8a) and (2.10)) equation (2.12) becomes a second-order ordinary differential equation for the determination of $S$. Once $S$ is determined the quantities $\Sigma_{r}$ and $\Sigma_{\theta}$ are given by (2.8a) and (2.10). The strains are determined by (2.14), the stresses by (2.3), and the displacements by (2.4).

The boundary conditions on equation (2.12) are nonlinear relations involving $S$ and $S^{\prime}$. In particular, the displacement condition (1.12) becomes (cf. (2.1b))

$$
1+\frac{\mu}{a}=\left.\sqrt{2 \varepsilon_{\theta}\left(\Sigma_{r}, \Sigma_{\theta}\right)+1}\right|_{r=a}
$$

while the stress condition (1.13) becomes (cf. (2.3))

$$
\sigma=\left.\frac{\Sigma_{r}}{\sqrt{2 \varepsilon_{\theta}\left(\Sigma_{r}, \Sigma_{\theta}\right)+1}}\right|_{r=a} .
$$

Of course both $\Sigma_{r}$ and $\Sigma_{\theta}$ are known functions of $S$ and $S^{\prime}$. In addition to (2.15) or (2.16) $S(r)$ should be finite at $r=0$.

3. Small strains. In this section we will specialize the exact theory described in Section 2 to the case of small strains. Thus in equation (2.12) we will replace $\sqrt{2 \varepsilon_{r}+1}$ and $\sqrt{2 \varepsilon_{\theta}+1}$ by $1+\varepsilon_{r}$ and $1+\varepsilon_{\theta}$ which are exact up to terms quadratic in $\varepsilon_{r}$ and $\varepsilon_{\theta}$. In any case (2.12) becomes

$$
1+\frac{d}{d r}\left(r \varepsilon_{\theta}\right)=\frac{m\left(1+\varepsilon_{r}\right)\left(S-F_{1}\right)}{\Sigma_{r}} .
$$

The appropriate constitutive laws in the small strain case are the Hooke's laws (1.10). Since the strains are assumed small the Hooke's laws can be written in terms of $\Sigma_{r}$ and $\Sigma_{\theta}$ (cf. (2.3)). In particular,

$$
\varepsilon_{r}=\frac{1}{E}\left(\Sigma_{r}-\nu \Sigma_{\theta}\right), \quad \varepsilon_{\theta}=\frac{1}{E}\left(\Sigma_{\theta}-\nu \Sigma_{r}\right) .
$$

These expressions are also exact up to quadratic terms in $\varepsilon_{r}$ and $\varepsilon_{\theta}$.

Combining (3.1) and (3.2) we find

$$
\begin{aligned}
1+\frac{d}{d r}\left(\frac{r(r S)^{\prime}}{E m}\right)= & \frac{m\left(S-F_{1}\right)}{\Sigma_{r}} \\
& +\frac{m\left(S-F_{1}\right)}{E}+\frac{\nu}{2 r E \Sigma_{r}}\left(\left(r^{2} F_{2}^{2}\right)^{\prime}+2 r\left(S-F_{1}\right)\left(r F_{1}\right)^{\prime}\right) .
\end{aligned}
$$

The notation is simplified if we introduce the change of variable

$$
T=S / E, \quad G_{1}=F_{1} / E, \quad G_{2}=F_{2} / E
$$


so that

$$
\Sigma_{r}=E \sqrt{G_{2}^{2}+\left(T-G_{1}\right)^{2}}
$$

and equation (3.3) becomes

$$
\begin{aligned}
1+\frac{d}{d r}\left(\frac{r(r T)^{\prime}}{m}\right) & =\frac{m\left(T-G_{1}\right)}{\sqrt{G_{2}^{2}+\left(T-G_{1}\right)^{2}}} \\
& +m\left(T-G_{1}\right)+\frac{\nu}{2 r \sqrt{G_{2}^{2}+\left(T-G_{1}\right)^{2}}}\left(\left(r^{2} G_{2}^{2}\right)^{\prime}+2 r\left(T-G_{1}\right)\left(r G_{1}\right)^{\prime}\right) .
\end{aligned}
$$

The plane circular membrane is a special case of (3.6). In particular if $z^{\prime}=0(m=1)$, which implies $G_{1}=0$ (cf. (1.7) or (1.8)), equation (3.6) becomes

$$
r^{2} T^{\prime \prime}+3 r T^{\prime}=\frac{T}{\sqrt{G_{2}^{2}+T^{2}}}-1+\frac{\nu\left(R^{2} G_{2}^{2}\right)^{\prime}}{2 r \sqrt{G_{2}^{2}+T^{2}}},
$$

where

$$
G_{2}=\frac{1}{E h r} \int_{0}^{r} \tau P(\tau) d \tau
$$

We note that if the last term on the right of (3.7) is neglected we obtain

$$
r^{2} T^{\prime \prime}+3 r T^{\prime}=\frac{T}{\sqrt{G_{2}^{2}+T^{2}}}-1 .
$$

In slightly different variables this is the theory proposed by Reissner for the plane circular membrane (cf. [6,7]). If it is assumed that $G_{2} / T \rightarrow 0$ as $G_{2} \rightarrow 0$ then for small $G_{2}$ equation (3.7) becomes

$$
r^{2} T^{\prime \prime}+3 r T^{\prime}=-\frac{G_{2}}{2 T^{2}} .
$$

This is the theory proposed by Föppl [5]. The relation between (3.10) and the exact equations for the plane circular membrane was described in [4]. The solutions of (3.9) and (3.10) have been discussed in [8-11].

4. The gravity case. In this section we will discuss the case in which the applied pressure is in the $z$ direction (cf. (1.7)). The equation (3.6) becomes

$$
1+\frac{d}{d r}\left(\frac{r(r T)^{\prime}}{m}\right)=\frac{m T}{\sqrt{G_{2}^{2}+T^{2}}}+m T+\frac{\nu\left(r^{2} G_{2}^{2}\right)^{\prime}}{2 r \sqrt{G_{2}^{2}+T^{2}}},
$$

where

$$
G_{2}=\frac{1}{E h r} \int_{0}^{r} \tau m P d \tau
$$


The boundary conditions on (4.1) are $|T(0)|<\infty$ and either the displacement condition (cf. (2.15))

$$
\left(\frac{(r T)^{\prime}}{m}-\nu \sqrt{G_{2}^{2}+T^{2}}\right)_{r=a}=\frac{\mu}{a}
$$

or the stress condition (cf. (2.16))

$$
\left(\frac{\sqrt{G_{2}^{2}+T^{2}}}{1+\frac{(r T)^{\prime}}{m} \nu \sqrt{G_{2}^{2}+T^{2}}}\right)_{r=a}=\frac{\sigma}{E} .
$$

One case of special interest is the zero pressure case, i.e., $G_{2}=0$. We assume the prescribed boundary condition (either $\sigma$ or $\mu$ ) is not zero. In this simple situation equation (4.1) reduces to the linear equation

$$
\frac{d}{d r}\left(\frac{(r T)^{\prime}}{m}\right)-m T=m-1
$$

We note that the solution of (4.5) is not zero nor does it approach zero as the boundary data approaches zero (unless $z^{\prime}=0$ ). Since $T \neq 0$ equation $(2.4 \mathrm{~b})$ implies that $z^{\prime}+w^{\prime}=0$ or equivalently $w=-z$, i.e., the deformed membrane is flat. Thus the solution of (4.5) determines the stress distribution in a membrane cap which has been deformed into a plane membrane.

It seems unlikely that a membrane cap would actually be deformed into a plane membrane by the application of arbitrarily small boundary stress $\sigma$ or boundary displacement $\mu$. However, this is the only angle-independent solution. Thus it is likely that the actual observed solution to this boundary value problem would be angle-dependent. Of course the verification of the existence of such solutions would rest on the study of a more general theory in which angular dependence is included.

If the applied pressure $G_{2} \neq 0$ and the boundary stress $\sigma$ or boundary displacement $\mu$ is sufficiently small then it is reasonable to expect that the actual deformation is independent of angle and it makes sense to study the solution of (4.1) in this case. We are interested in solutions of (4.1) which have the property that $w \rightarrow 0$ and $T \rightarrow 0$ as $G_{2} \rightarrow 0$ (i.e., as $P_{2} \rightarrow 0$ ). These are the axially symmetric solutions which approach the undeformed cap as $P_{2} \rightarrow 0$.

Assuming small strains, equation (2.4b) can be written

$$
z^{\prime}+w^{\prime}=-\frac{F_{2} m\left(1+\varepsilon_{r}\right)}{\sum_{r}}
$$

or, equivalently,

$$
z^{\prime}+w^{\prime}=-\frac{G_{2} m}{\sqrt{G_{2}^{2}+T^{2}}}\left(1+\frac{1}{E}\left(\Sigma_{r}-\nu \Sigma_{\theta}\right)\right) .
$$

$\Sigma_{r}$ and $\Sigma_{\theta}$ can be rewritten in terms of $T$ and $T^{\prime}$ so that

$$
z^{\prime}+w^{\prime}=-\frac{G_{2} m}{\sqrt{G_{2}^{2}+T^{2}}}\left(1+\sqrt{G_{2}^{2}+T^{2}}-\frac{\nu(r T)^{\prime}}{m}\right) .
$$


Equation (4.8) and the assumption that $T \rightarrow 0$ and $w \rightarrow 0$ as $G_{2} \rightarrow 0$ lead to the condition

$$
\lim _{G_{2} \rightarrow 0} \frac{G_{2}}{T}=-z^{\prime}
$$

It is convenient to rewrite (4.9) in the form

$$
\frac{G_{2}}{T}=-z^{\prime}+v
$$

where $v$ is small when $G_{2}$ is small.

In order to obtain an approximate theory for small values of $G_{2}$, we rewrite equation (4.1) in the form

$$
\frac{d}{d r}\left(\frac{r(r T)^{\prime}}{m}\right)-m T=\frac{m}{\sqrt{1+\left(\frac{G_{2}}{T}\right)^{2}}}-1+\frac{\nu\left(r G_{2}\right)^{\prime}}{\sqrt{1+\left(\frac{G_{2}}{T}\right)^{2}}}\left(\frac{G_{2}}{T}\right)
$$

or using (4.10)

$$
\frac{d}{d r}\left(\frac{r(r T)^{\prime}}{m}\right)-m T=\frac{m}{\sqrt{1+\left(v-z^{\prime}\right)^{2}}}-1+\frac{\nu\left(r G_{2}\right)^{\prime}}{\sqrt{1+\left(v-z^{\prime}\right)^{2}}}\left(v-z^{\prime}\right) .
$$

Since $v$ is a small quantity (when $G_{2}$ is small) the right side of (4.12) can be expanded in powers of $v$. Up to terms of second order in $v$ we have

$$
\begin{aligned}
\frac{d}{d r}\left(\frac{r(r T)^{\prime}}{m}\right)-m T= & -\frac{\nu z^{\prime}\left(r G_{2}\right)^{\prime}}{m} \\
& +\left(\frac{z^{\prime}}{m^{2}}+\frac{\nu\left(r G_{2}\right)^{\prime}}{m^{3}}\right) v+\left(\frac{2\left(z^{\prime}\right)^{2}-1}{2 m^{4}}+\frac{3 \nu z^{\prime}\left(r G_{2}\right)^{\prime}}{2 m^{5}}\right) v^{2}
\end{aligned}
$$

Using (4.10) equation (4.13) can be rewritten in terms of $T$. This equation is an approximate theory which applies when the applied pressure is small. It is not a shallow cap theory. Indeed no assumptions have been made on the magnitude of $z^{\prime}$. However, the plane membrane $\left(z^{\prime}=0\right)$ is a special case. If $z^{\prime}=0$ equation (4.13) reduces to

$$
r^{2} T^{\prime \prime}+3 r T^{\prime}=\nu\left(r G_{2}\right)^{\prime} \frac{G_{2}}{T}-\frac{1}{2} \frac{G_{2}^{2}}{T^{2}} .
$$

This equation is related to the Föppl equation for the plane circular membrane (cf. [5]). In fact it is of higher-order accuracy than the Föppl equation and reduces to the Föppl equation when $G_{2}$ is sufficiently small.

One case of particular interest is the shallow cap theory, i.e., $\left|z^{\prime}\right| \ll 1$. In this case equation (4.13) can be replaced by

$$
r^{2} T^{\prime \prime}+3 r T^{\prime}=-\nu z^{\prime}\left(r G_{2}\right)^{\prime}+\left(z^{\prime}+\nu\left(r G_{2}\right)^{\prime}\right) v-\frac{1}{2} v^{2} .
$$


Using (4.16) to replace $v$ in (4.15) we find

$$
r^{2} T^{\prime \prime}+3 r T^{\prime}=\frac{\left(z^{\prime}\right)^{2}}{2}+\nu\left(r G_{2}\right)^{\prime} \frac{G_{2}}{T}-\frac{1}{2}\left(\frac{G_{2}}{T}\right)^{2},
$$

where the forcing term $G_{2}$ is given by (cf. (4.2))

$$
G_{2}=\frac{1}{E h r} \int_{0}^{r} \tau P(\tau) d \tau
$$

when the membrane is shallow.

It remains to determine the appropriate boundary conditions for equation (4.13). There is no difficulty in showing that when $G_{2}$ is small the displacement boundary condition (4.3) becomes

$$
\left(\frac{(r T)^{\prime}}{m}-\nu m T\right)_{r=a}=\frac{\mu}{a}
$$

while the stress boundary condition (4.4) becomes

$$
(m T)_{r=a}=\frac{\sigma}{E} \text {. }
$$

In the shallow cap theory, i.e. equation (4.16), the two boundary conditions (4.18) and (4.19) became

$$
\begin{gathered}
a T^{\prime}(a)+(1-\nu) T(a)=\frac{\mu}{a}, \\
T(a)=\frac{\sigma}{E} .
\end{gathered}
$$

Once $T$ is determined the stresses and displacements are easily found. There is no difficulty in showing that when $G_{2}$ is small

$$
\begin{aligned}
& \sigma_{r}=E m T, \\
& \sigma_{\theta}=E \frac{(r T)^{\prime}}{m},
\end{aligned}
$$

and $u$ and $w$ are determined from

$$
\begin{aligned}
u & =r\left(\frac{(r T)^{\prime}}{m}-\nu m T\right), \\
w^{\prime} & =z^{\prime}\left(m T-\nu \frac{(r T)^{\prime}}{m}\right) .
\end{aligned}
$$

Of course in the shallow cap theory equations (4.22) and (4.23) are replaced by

$$
\begin{aligned}
\sigma_{r} & =E T, \\
\sigma_{\theta} & =E(r T)^{\prime}, \\
u & =r\left((r T)^{\prime}-\nu T\right), \\
w^{\prime} & =z^{\prime}\left(T-\nu(r T)^{\prime}\right) .
\end{aligned}
$$


As a case of special interest consider a membrane cap consisting of a section of a spherical surface of radius $R$. The equation of the cap is given by

$$
z=\sqrt{R^{2}-r^{2}}-\sqrt{R^{2}-a^{2}}, \quad 0 \leqslant r \leqslant a .
$$

Thus $z^{\prime}$ is given by

$$
z^{\prime}=\frac{-\gamma \rho}{\sqrt{1-\gamma^{2} \rho^{2}}}
$$

where

$$
\rho=r / a, \quad \gamma=a / R .
$$

If the cap is shallow then $\gamma \ll 1$ so that $z^{\prime}$ is approximated by

$$
z^{\prime}=-\gamma \rho .
$$

If we assume $P$ is constant there is no difficulty in showing that equation (4.16) reduces to

$$
\rho^{2} \frac{d^{2} T}{d \rho^{2}}+3 \rho \frac{d T}{d \rho}=\frac{\gamma^{2} \rho^{2}}{2}+\frac{\nu}{2} k^{3} \frac{\rho^{2}}{T}-\frac{k^{3}}{8} \frac{\rho^{2}}{T^{2}}, \quad 0 \leqslant \rho \leqslant 1
$$

where

$$
k^{3}=\frac{P^{2} a^{2}}{E^{2} h^{2}} .
$$

The displacement boundary condition is

$$
\left(\frac{d T}{d \rho}+(1-\nu) T\right)_{\rho=1}=\frac{\mu}{a},
$$

and the stress boundary condition is

$$
T(1)=\frac{\sigma}{E}
$$

In either case $T(\rho)$ should be finite at $\rho=0$. The stresses and displacements are determined from equations (4.24) and (4.25):

$$
\begin{gathered}
\sigma_{r}=E T, \\
\sigma_{\theta}=E \frac{d}{d \rho}(\rho T), \\
\frac{u}{a}=\rho\left(\frac{d}{d \rho}(\rho T)-\nu T\right), \\
\frac{1}{a} \frac{d w}{d \rho}=-\gamma \rho\left(T-\nu \frac{d}{d \rho}(\rho T)\right) .
\end{gathered}
$$

5. The normal pressure case. In this section we will consider the case in which the pressure is normal to the undeformed surface. The governing equation is (3.6) where

$$
G_{1}=\frac{-1}{E h r} \int_{0}^{r} \tau z^{\prime} P d \tau, \quad G_{2}=\frac{1}{E h r} \int_{0}^{r} \tau P d \tau .
$$


Just as in the previous section we wish to study solutions which have the property that both $T \rightarrow 0$ and $w \rightarrow 0$ as $P \rightarrow 0\left(G_{1} \rightarrow 0\right.$ and $\left.G_{2} \rightarrow 0\right)$. It is a consequence of $(2.4 \mathrm{~b})$, the assumption of small strains, and (3.2) that

$$
z^{\prime}+w^{\prime}=\frac{-G_{2} m}{\sqrt{G_{2}^{2}+\left(T-G_{1}\right)^{2}}}\left(1+\sqrt{G_{2}^{2}+\left(T-G_{1}\right)^{2}}-\frac{\nu(r T)^{\prime}}{m}\right)
$$

or equivalently,

$$
z^{\prime}+w^{\prime}=\frac{\left(\frac{G_{2}}{T-G_{1}}\right)}{\sqrt{1+\left(\frac{G_{2}}{T-G_{1}}\right)^{2}}}\left(1+\left(T-G_{1}\right) \sqrt{1+\left(\frac{G_{2}}{T-G_{1}}\right)^{2}}-\frac{\nu(r T)^{\prime}}{m}\right)
$$

As $G_{1} \rightarrow 0$ and $G_{2} \rightarrow 0$ we require that $T \rightarrow 0$ and $w \rightarrow 0$. It follows from (5.3) that

$$
\lim _{P \rightarrow 0} \frac{G_{2}}{T-G_{1}}=-z^{\prime}
$$

Thus we write

$$
\frac{G_{2}}{T-G_{1}}=-z^{\prime}+v
$$

where $v$ is small when $P$ is small. Using (5.5) we can rewrite equation (3.6) as

$$
\begin{aligned}
\frac{d}{d r}\left(\frac{r(r T)^{\prime}}{m}\right)-m\left(T-G_{1}\right)= & \frac{m}{\sqrt{1+\left(v-z^{\prime}\right)^{2}}}-1 \\
& \quad+\frac{\nu}{\sqrt{1+\left(v-z^{\prime}\right)^{2}}}\left(\left(r G_{2}\right)^{\prime}\left(v-z^{\prime}\right)+\left(r G_{1}\right)^{\prime}\right) .
\end{aligned}
$$

It is a consequence of equation (5.1) that equation (5.6) can be written

$$
\begin{aligned}
& \frac{d}{d r}\left(\frac{r(r T)^{\prime}}{m}\right)-m\left(T-G_{1}\right)=\frac{m}{\sqrt{1+\left(v-z^{\prime}\right)^{2}}}-1 \\
& \frac{-2 v z^{\prime}\left(r G_{2}\right)^{\prime}}{\sqrt{1+\left(v-z^{\prime}\right)^{2}}}+\frac{\nu\left(r G_{2}\right)^{\prime}}{\sqrt{1+\left(v-z^{\prime}\right)^{2}}} v,
\end{aligned}
$$

where we have used the fact that (cf. (5.1))

$$
\left(r G_{1}\right)^{\prime}=-z^{\prime}\left(r G_{2}\right)^{\prime} .
$$


Since $v$ is a small quantity when $P$ is small, we will only retain terms up to second order in $v$. Thus equation (5.7) can be replaced by

$$
\begin{aligned}
\frac{d}{d r}\left(\frac{r(r T)^{\prime}}{m}\right)-m\left(T-G_{1}\right)= & -\frac{2 \nu z^{\prime}\left(r G_{2}\right)^{\prime}}{m} \\
& +\left(\frac{z^{\prime}}{m^{2}}+\frac{\left(1-\left(z^{\prime}\right)^{2}\right) \nu\left(r G_{2}\right)^{\prime}}{m^{3}}\right) v \\
& +\left(\frac{2\left(z^{\prime}\right)^{2}-1}{2 m^{4}}+\frac{3\left(z^{\prime}\right)^{3} \nu\left(r G_{2}\right)^{\prime}}{m^{5}}\right) v^{2}
\end{aligned}
$$

Equation (5.9) can be rewritten entirely in terms of $T$ using (5.5).

The boundary conditions on (5.9) are easily determined from (2.15) or (2.16) and the assumption of small strains. The displacement condition becomes

$$
\left(\frac{(r T)^{\prime}}{m}-\nu m\left(T-G_{1}\right)\right)_{r=a}=\frac{\mu}{a} .
$$

The stress condition is given by

$$
\left(m\left(T-G_{1}\right)\right)_{r=a}=\frac{\sigma}{E} .
$$

The condition at the center remains unchanged, i.e., $|T(0)|<\infty$.

Once $T$ is determined the stresses and displacements are easily found. Thus we find (cf. $(2.8 \mathrm{a}),(2.10))$

$$
\begin{aligned}
\sigma_{r} & =E m\left(T-G_{1}\right), \\
\sigma_{\theta} & =\frac{E(r T)^{\prime}}{m} .
\end{aligned}
$$

The displacements are given by

$$
\begin{gathered}
u=r\left(\frac{(r T)^{\prime}}{m}-\nu m\left(T-G_{1}\right)\right), \\
w^{\prime}=z^{\prime}\left(m\left(T-G_{1}\right)-\nu \frac{(r T)^{\prime}}{m}\right) .
\end{gathered}
$$

Just as in Section 4 we note that the above theory is not a shallow cap theory. However, if $z^{\prime}=0$, equation (5.9) reduces to equation (4.14) - the equation for the plane membrane. If $\left|z^{\prime}\right| \ll 1$, i.e. the shallow cap theory, equation (5.9) can be replaced by

$$
\frac{d}{d r}\left(r(r T)^{\prime}\right)-\left(T-G_{1}\right)=-2 \nu z^{\prime}\left(r G_{2}\right)^{\prime}+\left(z^{\prime}+\nu\left(r G_{2}\right)^{\prime}\right) v-\frac{v^{2}}{2}
$$

or, using (5.5),

$$
\begin{aligned}
\frac{d}{d r}\left(r(r T)^{\prime}\right)-\left(T-G_{1}\right)=\frac{\left(z^{\prime}\right)^{2}}{2}-\nu z^{\prime}\left(r G_{2}\right)^{\prime} & \\
& +\nu\left(r G_{2}\right)^{\prime} \frac{G_{2}}{T-G_{1}}-\frac{1}{2}\left(\frac{G_{2}}{T-G_{1}}\right)^{2} .
\end{aligned}
$$


Equation (5.15) is simplified if we introduce a new dependent variable

$$
Y=T-G_{1} \text {. }
$$

In this new variable, equation (5.15) becomes

$$
r^{2} Y^{\prime \prime}+3 r Y^{\prime}=\frac{\left(z^{\prime}\right)^{2}}{2}-\nu z^{\prime}\left(r G_{2}\right)^{\prime}-\frac{d}{d r} r\left(r G_{1}\right)^{\prime}+\nu\left(r G_{2}\right)^{\prime} \frac{G_{2}}{Y}-\frac{1}{2}\left(\frac{G_{2}}{Y}\right)^{2}
$$

The boundary conditions for the shallow cap theory are determined from (5.10) or (5.11). In particular, the displacement condition becomes

$$
\left((r Y)^{\prime}+\nu Y\right)_{r=a}=\frac{\mu}{a}-\left(r G_{1}\right)_{r=a}^{\prime} .
$$

The stress condition is given by

$$
Y(a)=\frac{\sigma}{E} \text {. }
$$

The center condition is $|Y(0)|<\infty$. The stresses and displacements are (cf. (5.12) and (5.13))

$$
\begin{aligned}
\sigma_{r} & =E Y \\
\sigma_{\theta} & =E\left((r Y)^{\prime}+\left(r G_{1}\right)^{\prime}\right), \\
u & =r\left((r Y)^{\prime}-\nu Y\right)+r\left(r G_{1}\right)^{\prime} \\
w^{\prime} & =z^{\prime}\left(Y-\nu(r Y)^{\prime}\right)-\nu z^{\prime}\left(r G_{1}\right)^{\prime} .
\end{aligned}
$$

As in Section 4 we consider the special case of the shallow spherical cap under constant pressure. In the notation of Section 4 (cf. (4.26)-(4.29))

$$
G_{1}=\frac{\gamma P a \rho^{2}}{3 E h}, \quad G_{2}=\frac{P a \rho}{2 E h}, \quad 0 \leqslant \rho \leqslant 1 .
$$

The differential equation (5.17) becomes

$$
\rho^{2} \frac{d^{2} Y}{d \rho^{2}}+3 \rho \frac{d Y}{d \rho}=\left(\frac{\gamma^{2}}{2}-(3-\nu) \gamma k^{3 / 2}\right) \rho^{2}+\frac{\nu k^{3}}{2} \frac{\rho^{2}}{Y}-\frac{k^{3}}{8} \frac{\rho^{2}}{Y^{2}}
$$

where $k$ is defined in (4.31). The boundary conditions on (5.23) are easily found from (5.18) and (5.19). The displacement condition is

$$
\left(\frac{d Y}{d \rho}+(1-\nu) Y\right)_{\rho=1}=\frac{\mu}{a}+\gamma k^{3 / 2}
$$

and the stress condition is

$$
Y(1)=\frac{\sigma}{E} .
$$

Of course the condition at the center remains $|Y(0)|<\infty$. The stresses are

$$
\begin{gathered}
\sigma_{r}(\rho)=E Y(\rho), \\
\sigma_{\theta}(\rho)=E\left(\frac{d}{d \rho}(\rho Y)+\gamma k^{3 / 2} \rho^{2}\right) .
\end{gathered}
$$


The displacements are determined by

$$
\begin{gathered}
\frac{u}{a}=\rho\left(\rho \frac{d Y}{d \rho}+(1-\nu) Y+\gamma k^{3 / 2} \rho^{2}\right), \\
\frac{1}{a} \frac{d w}{d \rho}=\gamma \rho\left(\nu \rho \frac{d Y}{d \rho}-(1-\nu) Y\right)+\nu \gamma^{2} k^{3 / 2} \rho^{3} .
\end{gathered}
$$

6. Appendix. The exact equations. In this section we will derive the strain displacement equations (1.4) and equilibrium equations (1.6). In order to determine the strains we note that, as a result of the deformation described by (1.2) and (1.3), an element of length $d s$ before deformation is transformed into an element of length $d s^{*}$ after deformation where

$$
\begin{aligned}
(d s)^{2} & =d \mathbf{R} \cdot d \mathbf{R}=\left(1+\left(z^{\prime}\right)^{2}\right)(d r)^{2}+r^{2}(d \theta)^{2}, \\
\left(d s^{*}\right)^{2} & =d \mathbf{R}^{*} \cdot d \mathbf{R}^{*} \\
& =\frac{\left(1+u^{\prime}\right)^{2}+\left(z^{\prime}+w^{\prime}\right)^{2}}{1+\left(z^{\prime}\right)^{2}}\left(1+\left(z^{\prime}\right)^{2}\right)(d r)^{2}+\left(1+\frac{u}{r}\right)^{2} r^{2}(d \theta)^{2} .
\end{aligned}
$$

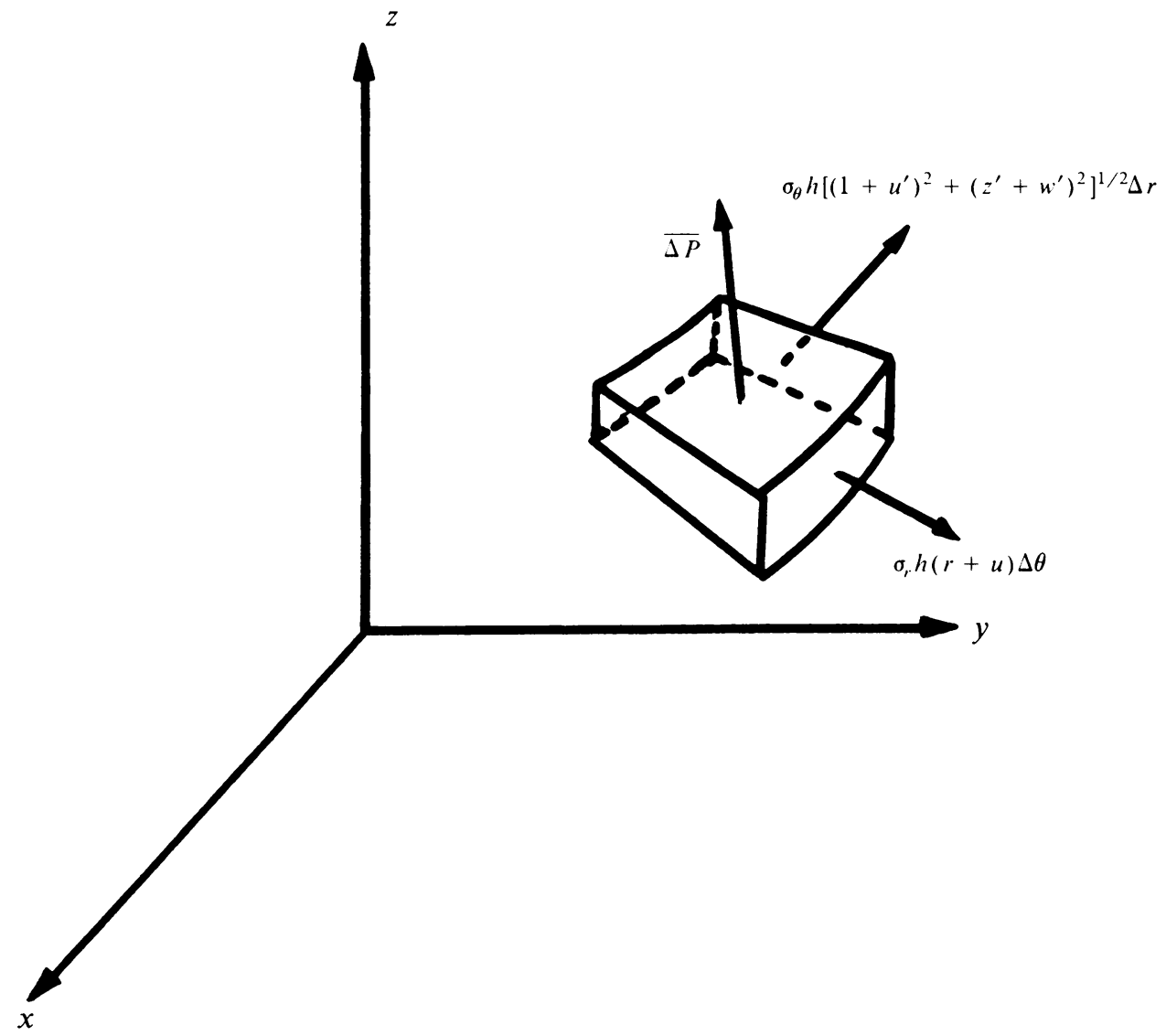

Fig. 1. 
The strains are determined by

$$
\left(d s^{*}\right)^{2}-(d s)^{2}=2 \varepsilon_{r}\left(1+\left(z^{\prime}\right)^{2}\right) d r^{2}+2 \varepsilon_{\theta} r^{2}(d \theta)^{2} .
$$

It follows that $\varepsilon_{r}$ and $\varepsilon_{\theta}$ are given by (1.4a) and (1.4b).

In Fig. 1 we have drawn an element of the deformed membrane and indicated the magnitude of the forces on the positive faces. The unit normal to the face $r=$ constant is

$$
\frac{\left(1+u^{\prime}\right) \mathbf{e}_{r}+\left(z^{\prime}+w^{\prime}\right) \mathbf{k}}{\sqrt{\left(1+u^{\prime}\right)^{2}+\left(z^{\prime}+w^{\prime}\right)^{2}}}
$$

and the unit normal to the face $\theta=$ constant is $\mathbf{e}_{\theta}$. Thus equilibrium requires

$$
\begin{aligned}
& \left.\frac{\sigma_{r} h(r+u)\left[\left(1+u^{\prime}\right) \mathbf{e}_{r}+\left(z^{\prime}+w^{\prime}\right) \mathbf{k}\right]}{\left[\left(1+u^{\prime}\right)^{2}+\left(z^{\prime}+w^{\prime}\right)^{2}\right]^{1 / 2}} \Delta \theta\right|_{r} ^{r+\Delta r} \\
& +\left.\sigma_{\theta} h\left[\left(1+u^{\prime}\right)^{2}+\left(z^{\prime}+w^{\prime}\right)^{2}\right]^{1 / 2} \mathbf{e}_{\theta} \Delta r\right|_{\theta} ^{\theta+\Delta \theta}+\overline{\Delta P}=0 .
\end{aligned}
$$

We consider two choices for $\overline{\Delta P}$. In either case $\overline{\Delta P}$ is the force per unit undeformed area and its direction is in either the $z$ direction or normal to the undeformed surface. The area of the element depicted in Fig. 1 before deformation is

$$
\Delta A=\left|\frac{\partial \mathbf{R}}{\partial r} \times \frac{\partial \mathbf{R}}{\partial \theta}\right| \Delta r \Delta \theta .
$$

This quantity is easily calculated from (1.2). Thus

$$
\frac{\partial \mathbf{R}}{\partial r} \times \frac{\partial \mathbf{R}}{\partial \theta}=\left(\mathbf{e}_{r}+z^{\prime} \mathbf{k}\right) \times\left(r \mathbf{e}_{\theta}\right)=r\left(-z^{\prime} \mathbf{e}_{r}+\mathbf{k}\right), .
$$

so that

$$
\Delta A=r \sqrt{1+\left(z^{\prime}\right)^{2}} \Delta r \Delta \theta=r m \Delta r \Delta \theta .
$$

If $\overline{\Delta P}$ is the $z$ direction then

$$
\overline{\Delta P}=(r m P \Delta r \Delta \theta) \mathbf{k}
$$

where $P$ is the force per unit undeformed area. The unit normal to the undeformed surface is obtained from (6.7). Thus we find

$$
\bar{n}=\frac{-z^{\prime} \mathbf{e}_{r}+\mathbf{k}}{m} .
$$

It follows that in the normal pressure case

$$
\begin{aligned}
\overline{\Delta P} & =(r m P \Delta r \Delta \theta)\left(\frac{-z^{\prime} \mathbf{e}_{r}+\mathbf{k}}{m}\right) \\
& =r P\left(-z^{\prime} \mathbf{e}_{r}+\mathbf{k}\right) \Delta r \Delta \theta .
\end{aligned}
$$

In either the gravity case (6.9) or the normal pressure case (6.11) we can write

$$
\overline{\Delta P}=\left(P_{1} \mathbf{e}_{r}+P_{2} \mathbf{k}\right) \Delta r \Delta \theta,
$$

where $P_{1}$ and $P_{2}$ are given by either (1.8) or (1.9). 
The equilibrium equations are obtained by dividing equation (6.5) by $h \Delta r \Delta \theta$ and taking the limit as $\Delta r \rightarrow 0$ and $\Delta \theta \rightarrow 0$. We find

$$
\begin{aligned}
& \frac{d}{d r}\left\{\frac{\sigma_{r}(r+u)\left[\left(1+u^{\prime}\right) \mathbf{e}_{r}+\left(z^{\prime}+w^{\prime}\right) \mathbf{k}\right]}{\left[\left(1-u^{\prime}\right)^{2}+\left(z^{\prime}+w^{\prime}\right)^{2}\right]^{1 / 2}}\right\} \\
& \quad+\sigma_{\theta}\left[\left(1+u^{\prime}\right)^{2}+\left(z^{\prime}+w^{\prime}\right)^{2}\right]^{1 / 2} \mathbf{e}_{r}+\frac{P_{1}}{h} \mathbf{e}_{r}+\frac{P_{2}}{h} \mathbf{k}=0 .
\end{aligned}
$$

The equations (1.6) are an immediate consequence of (6.13).

\section{REFERENCES}

[1] E. Bromberg and J. J. Stoker, Non-linear theory of curved elastic sheets, Quart. Appl. Math. 3, 246-265 $(1945 / 46)$

[2] E. Reissner, Rotationally symmetric problems in the theory of thin elastic shells, 3rd U.S. Natn. Congr. of Applied Mechanics, 59-69, 1958

[3] M. A. Goldberg, An iterative solution for rotationally symmetric non-linear membrane problems, Int. J. Non-linear Mechs. 1 169-178 (1966)

[4] R. W. Dickey. The nonlinear circular membrane under a vertical force, Quart. Appl. Math. 41, 331-338 (1983)

[5] A. Föppl, Vorlesungen über technische Mechanik, Bd. 5, Leipzig: G. Teubner, 1907

[6] E. Reissner, On axisymmetric deformation of thin shells of revolution, Proc. Symp. Appl. Math. 3, 27-52 (1950)

[7] H. J. Weinitschke, On axisymmetric deformation of nonlinear elastic membranes, Mechanics Today 5. 523-542. Ed. S. Nemat-Nasser, Oxford and New York: Pergamon Press (1980)

[8] H. Hencky, Üher den Spannungszustand in kreisrunden Platten, Z. Math. Phys. 63, 311-317 (1915)

[9] R. W. Dickey, The plane circular elastic surface under normal pressure, Arch. Rat. Mech. Anal. 26, 219-236 (1967)

[10] A. J. Callegari and E. L. Reiss, Non-linear houndary value problems for the circular membrane, Arch. Rat. Mech. Anal. 31, 390-400 (1968)

[11] H. J. Weinitschke, On finite displacement of circular elastic membranes, Institute of Appl. Math. and Statistics, Univ. British Columbia. Vancouver, Canada, Tech. Rep. No. 85-7, 1985 